\title{
Problemas de resistencia en Streptococcus pyogenes
}

\author{
ROSSANNA CAMPONOVO C.*
}

\section{ANTIMICROBIAL RESISTANCE IN Streptococcus pyogenes}

Streptococcus pyogenes resistance problems are refered to macrolides resistance which occurs by two mechanisms: modification of the target site in the ribosomes, $M L S_{B}$ resistance codified by gen erm and associated with resistance to lincosamines and streptogramin $B$, or by an active efflux mechanism codified by gen mef which confers resistance to macrolides only, this being the most frequently described in this specie.

In Chile Streptococcus pyogenes strains resistant to macrolides have been isolated since 1994, with rates of 7\% in last six years at the Metropolitan area.

Key words: Macrolide resistance, Streptococcus pyogenes.

La clasificación de los estreptococos ha tenido últimamente grandes cambios basados en estudios taxonómicos moleculares del género Streptococcus lo que llevó a que enterococo y lactococo tengan actualmente sus propios géneros, Enterococcus y Lactococcus respectivamente.

En los estreptococos $\beta$ hemolíticos se conoce ahora que especies no relacionadas pueden producir igual antígeno de Lancefield y por el contrario, cepas genéticamente relacionadas a nivel de especie presenten antígenos de Lancefield heterogéneos.

Actualmente existen los Streptococcus $\beta$ hemolíticos A, B, C, F, G y no agrupable, de acuerdo al antígeno de Lancefield, de los cuales los grupos A, C y G pueden ser divididos en dos según el tamaño de la colonia: colonia grande $(>0,5 \mathrm{~mm} \varnothing)$ y colonia pequeña $(<0,5 \mathrm{~mm}$ $\varnothing)$. Los formadores de colonia grande son estreptococos "piogénicos" con una variedad de mecanismos efectivos de virulencia que se traducen en su patogenicidad, mientras que las cepas formadoras de colonias pequeñas son genéticamente distintas, y habitualmente forman parte de la flora microbiana comensal, aunque pueden aislarse ocasionalmente de infecciones como abscesos. Estas últimas pertenecen al grupo especie Streptococcus milleri o Streptococcus anginosus que mayoritariamente agrupa a Streptococcus $\alpha$ hemolíticos o no hemolíticos.

\section{Identificación}

Para lograr una correcta identificación de Streptococcus $\beta$ hemolíticos en el laboratorio se debe contar con las siguientes pruebas: $:^{1,2}$

- susceptibilidad a bacitracina

- pirrolidonil aminopeptidasa(PYR)

- test de Voges Proskauer(VP)

- test de CAMP

- test de aglutinación para antígeno de Lancefield.

\footnotetext{
* Laboratorio de Microbiología, Integramedica.
} 
Problema de resistencia en Streptococcus pyogenes - $R$. Camponovo $C$.

Tabla 1. Caracteres diferenciales de Streptococcus $\beta$ hemolíticos

\begin{tabular}{lllll}
\hline $\begin{array}{l}\text { Grupo } \\
\text { Lancefield }\end{array}$ & $\begin{array}{l}\text { Tamaño de la } \\
\text { colonia }\end{array}$ & PYR* & VP* & CAMP* \\
\hline A & grande & + & - & - \\
A & pequeña & - & + & - \\
B & grande & - & - & + \\
C & pequeña & - & + & - \\
C & pequeña & - & + & - \\
F & grande & - & - & - \\
G & pequeña & - & + & - \\
G & pequeña & - & + & - \\
\hline
\end{tabular}

PYR: pirrolinodil arilamidasa VP: Voges Proskauer CAMP: acróstico de los autores referencia 3).

Estas pruebas nos permiten identificar el grupo y diferenciar las cepas piogénicas de las pertenecientes al grupo S. milleri. (Tabla 1). En la identificación de $S$. pyogenes, la susceptibilidad a bacitracina ampliamente usada en nuestros laboratorios, es un diagnóstico presuntivo, de alta sensibilidad pero una baja especificidad (100 y 40,7\%, respectivamente) ya que estreptococos de los grupos B, C y G pueden ser susceptibles. Sí permite diferenciar $S$. pyogenes de cepas de colonias pequeñas del grupo A.

\section{Susceptibilidad de Streptococcus pyogenes a penicilina}

A diferencia de otras cocáceas Gram positivas, como Staphylococcus y Streptococus pneumoniae, $S$. pyogenes se ha mantenido susceptible a lo largo del tiempo a concentraciones muy bajas de penicilina, siendo éste el fármaco de elección; a pesar de su amplio uso por más de 50 años para el tratamiento de infecciones por este agente, no se han descrito hasta ahora cepas resistentes. La estabilidad en la susceptibilidad se observa claramente en un trabajo que comparó la CIM de 133 cepas aisladas entre los años 1917 y 1996, incluyendo cepas de la colección original de Lancefield y cepas representativas del mundo, en las cuales la $\mathrm{CIM}_{90}$ fue de $0,031 \mu \mathrm{g} / \mathrm{ml}$ para todas ellas ${ }^{6}$. Esta misma sensibilidad fue observada en 88 cepas aisladas entre 1996 y 1997 en Santiago por el Laboratorio Clínico Integramedica siendo la $\mathrm{CIM}_{90}$ también de $0,03 \mu \mathrm{g} / \mathrm{ml}$.
La falla en el tratamiento con penicilina en cuadros estreptocóccicos se ha atribuido a la presencia de otras bacterias productoras de $\beta$ lactamasa, especialmente en infecciones faríngeas que inactivarían la penicilina, y a un pobre cumplimiento de tratamiento por parte del paciente. El fenómeno de tolerancia, en que la cepa es inhibida pero la actividad bactericida del fármaco es reducida, ha sido descrita, pero no está claro su trascendencia clínica.

\section{Resistencia de Streptococcus pyogenes a macrólidos}

En 1974 se aislaron en Japón las primeras cepas resistentes a macrólidos y de ahí han sido descritas ampliamente en la literatura, en la mayoría de las áreas ha permanecido en baja frecuencia de resistencia, pero en países como España y Finlandia ha alcanzado porcentajes superiores a $30 \%$, lo que en varios trabajos se ha correlacionado con un mayor uso de macrólidos. En Japón se redujo el porcentaje de resistencia de $22 \%$ en 1981 a $1 \%$ en 1990 , lo que ha sido atribuido a una disminución del uso de macrólidos durante ese tiempo.

En nuestro país a partir de la decada del 90 se han aislado cepas resistentes a macrólidos. En el Laboratorio Clínico de la Pontificia Universidad Católica fueron aisladas entre 1990 y 1998, 594 cepas de $S$ pyogenes, encontrándose 32 cepas $(7,2 \%)$ resistentes. En el Laboratorio Clínico Integramédica se aislaron 477 cepas entre 1996 y 2000 encontrándose 29 cepas $(6,1 \%)$ resistentes. 


\section{Mecanismos de resistencia a macrólidos}

En $S$. pyogenes se describen dos mecanismos de resistencia a macrólidos: modificación del sitio de sitio blanco y eflujo activo ${ }^{4}$.

- Modificación del sitio blanco. Este mecanismo se produce por la adquisición de un gen erm (erythromycin ribosome methylase) que codifica una enzima que $\mathrm{N}^{6}$-dimetila un residuo específico de adenina en el 23SrARN, produciendo un cambio conformacional en el ribosoma que disminuye la afinidad a macrólidos, lincosamidas y streptogramina $\mathrm{B}$, fármacos químicamente distintos pero con similar mecanismo y sitio de acción. Esta resistencia cruzada se conoce como resistencia fenotípica $\mathrm{MLS}_{\mathrm{B}}$ y se expresa en forma constitutiva o inducible.

- Eflujo activo. Este mecanismo se produce por la presencia de un gen mef (macrolide efflux) que codifica la síntesis de una bomba que media el eflujo en forma activa ${ }^{7}$. Este eflujo activo sólo confiere resistencia a macrólidos por lo que se conoce como resistencia fenotípica M. Es el mecanismo de resistencia más frecuentemente descrito en $S$. pyogenes, lo que concuerda también con lo encontrado en cepas chilenas, ya que de las 29 cepas resistentes aisladas en el Laboratorio Integramédica $27(93,1 \%)$ correspondían a este fenotipo y de las 32 cepas resistentes aisladas en Laboratorio Clínico de la Pontificia Universidad Católica 28 $(87,5 \%)$, correspondían a este fenotipo detectándose en ellas la presencia del gen mef mediante amplificación con RPC. ${ }^{5}$

\section{Estudio de susceptibilidad de Streptococcus pyogenes en el laboratorio clínico}

En el laboratorio asistencial podemos detectar los tres fenotipos diferentes descritos en $S$. pyogenes resistentes a eritromicina de acuerdo a su susceptibilidad a clindamicina: susceptibles, de resistencia inducible y de resistencia constitutiva. ${ }^{4}$

\section{Método}

Se realiza antibiograma por método de difusión en disco en medio de agar Mueller Hinton con 5\% de sangre de cordero. Se prepara un inóculo con suspensión directa de colonias y se incuba a $35^{\circ} \mathrm{C}$ en atmósfera con $5 \% \quad \mathrm{CO}_{2}$ durante 20 a 24 horas de acuerdo a las normas de NCCLS para Streptococcus spp diferentes que Streptococccus pneumoniae.

Para detectar los diferentes fenotipos de resistencia se usa el llamado método de doble disco que consiste en colocar los sensidiscos de eritromicina $(15 \mu \mathrm{g})$ y clindamicina $(2 \mu \mathrm{g})$ a $16 \mathrm{~mm}$ de distancia de borde a borde para favorecer la inducción de resistencia a clindamicina, si ese mecanismo está presente. De esta manera obtenemos un patrón de antibiograma característico para cada fenotipo.

El fenotipo $\mathrm{M}$ confiere sólo resistencia a eritromicina, y entonces la clindamicina presenta un halo de inhibición redondo y simétrico. El fenotipo $\mathrm{MLS}_{\mathrm{B}}$ constitutivo otorga resistencia a eritromicina y clindamicina, con 2 halos pequeños y el fenotipo $\mathrm{MLS}_{\mathrm{B}}$ inducible dará un halo de inhibición pequeño de resistencia a eritromicina y un halo asimétrico a clindamicina con un aplanamiento de su halo hacia el lado de la eritromicina con lo que se demuestra que eritromicina indujo la expresión de la resistencia a clindamicina permitiendo detectarla en el laboratorio clínico (Figura 1)

Este método es más sensible y rápido para detectar y caracterizar las cepas resistentes a macrólidos que los métodos de dilución donde clindamicina puede aparecer falsamente sensible a las 24 horas de incubación y mostrar su resistencia a las 48 horas cuando corresponde al fenotipo $\mathrm{MLS}_{\mathrm{B}}$ inducible.

\section{RESUMEN}

Los problemas de resistencia de Streptococcus pyogenes son relativos a la adquisición de resistencia a macrólidos lo cual ocurre mediante dos mecanismos: modificación del sitio de acción en el ribosoma, resistencia tipo $\mathrm{MLS}_{\mathrm{B}}$ codificada por el gen erm que se asocia con resistencia a lincosamidas y streptogramina B, 


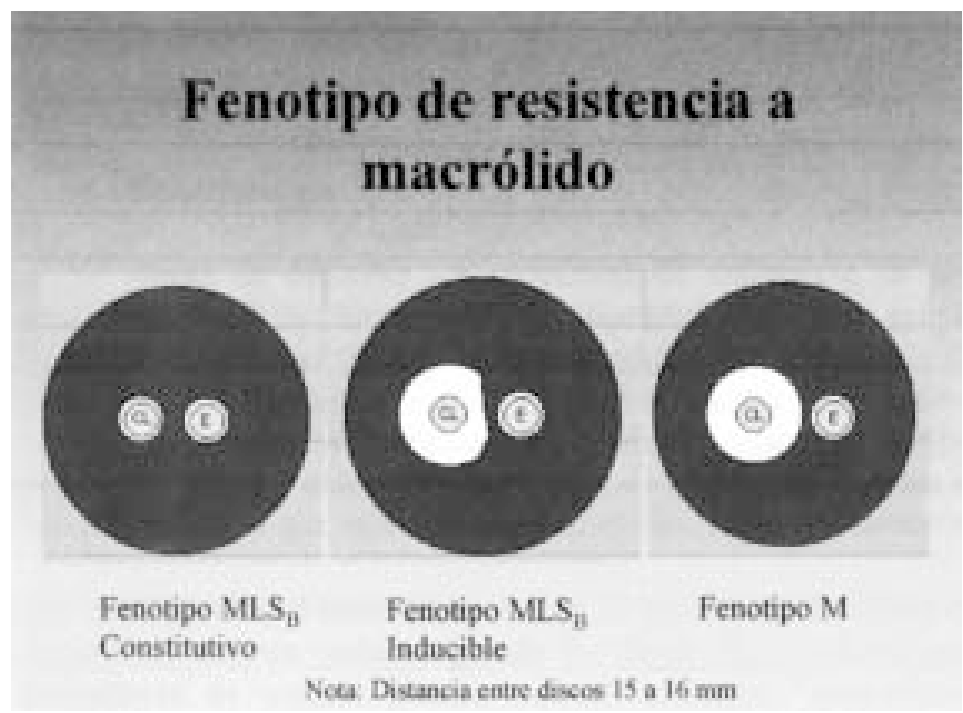

Figura 1. Representación de la expresión fenotípica de la resistencia a macrólidos en Streptococcus pyogenes

y por eflujo activo codificado por gen mef con resistencia sólo a macrólidos, siendo éste el mecanismo más frecuentemente encontrado en este microorganismo.

En Chile se han aislado cepas de Streptococcus pyogenes resistentes a macrólidos desde 1994, con porcentaje de resistencia promedio en los últimos seis años de $7 \%$ en el área Metropolitana.

\section{LECTURAS RECOMENDADAS}

1.- RUOFF K, WHILEY R, BEIGHTON D. Streptococcus. In: Murray PR, Baron EJ, Pfaller MA, Tenover FC, Yolken RH. Manual of Clinical Microbiology $7^{\text {th }}$ Edition, 1999. ASM, Washington DC, pp: 283-96.

2.- GIGLIO M, ULLOA M, ROBLES M et al. Uso de bacitracina, L-piroglutamil amilaridasa y aglutinación en el diagnóstico de Streptococcus $\beta$ - hemolítico. Rev Chil Infect 1998; 15(1): 39-44.
3.- CHRISTIE R, ATKINS N E, MUNCH-PETERSEN E. A note on a lytic phenomenon shown by group B streptococci. Aust J Exp Biol Med Sci 1944; 23: 193 7.

4.- QUINTILIANI R, SAHM D, COURVALIN P. Mechanisms of Resistance to Antimicrobial Agents. In: Murray PR, Baron EJ, Pfaller MA, Tenover FC, Yolken RH. Manual of Clinical Microbiology $7^{\text {th }}$ Edition, 1999. ASM, Washington, DC , pp: 1505-25.

5.- PALAVECINO E, RIEDEL I, BERRIOS $X$ et al Prevalence and mechanisms of macrolide resistance in Streptococcus pyogenes in Santiago, Chile. Antimicrob Agents Chemother 2001; 45(1): 339-41.

6.- MACRIS M, HARTMAN N, MURRAY B et al. Studies of the continuing susceptibility of group A streptococcal strains to penicillin during eight decades. Pediatr Infect Dis J 1998;17(5): 377-81

7.- SUTCLIFFE J, TAIT-KAMRADT A, WONDRACK L. Streptococcus pneumoniae and Streptococcus pyogenes resistant to macrolides but sensitive to clindamycin: a common resistance pattern mediated by an efflux system. Antimicrob Agents Chemother 1996 Aug; 40(8):1817-24.

Correspondencia a:

Rossanna Camponovo C.

Email: rcamponovo@terra.cl 